PROCEEDINGS OF THE

AMERICAN MATHEMATICAL SOCIETY

Volume 127, Number 9, Pages 2569-2572

S 0002-9939(99)05309-5

Article electronically published on May 17, 1999

\title{
ON FREE SUBGROUPS OF UNITS OF RINGS
}

\author{
A. SALWA \\ (Communicated by Ronald M. Solomon)
}

\begin{abstract}
We prove that if $a^{2}=b^{2}=0$ for elements $a, b$ of a ring $R$ of characteristic zero and $a b$ is not nilpotent, then there exists $m \in \mathbf{N}$ such that the group generated by $1+m a$ and $1+m b$ is free nonabelian. This is used to prove that a noncommutative positive-definite algebra with involution over an uncountable field contains a free nonabelian subsemigroup.
\end{abstract}

Consider the following general problem: when does a ring $R$ (of characteristic zero) contain a free nonabelian subgroup, or subsemigroup, and how does one construct such free objects? In [2] Klein proved that if $R$ is a noncommutative domain and the center of $R$ is uncountable, then $R$ contains free nonabelian subsemigroups. It is still an open problem whether every noncommutative division ring $R$ contains a free nonabelian subgroup. In this paper we are interested in the case when $R$ contains nilpotent elements. In [3] Marciniak and Sehgal proved that the group generated by a nontrivial bicyclic unit of an integral group ring $\mathbf{Z}[G]$ and by its conjugate (under the natural involution of $\mathbf{Z}[G]$ ) must be free. Unexpectedly, this has led to a new insight into the structure of unit groups and in particular to new simple proofs of several results. Bicyclic units have the form $1+a$, where $a^{2}=0$. This result was generalized to algebras over $\mathbf{C}$ with involution $*$ and endowed with a Hermitian inner product which is compatible with $*$, and to subgroups generated by $1+a$ and $1+a^{*}$, where $a^{2}=0$ [4]. In this paper we investigate the general case of subgroups generated by elements $1+a$ and $1+b, a^{2}=b^{2}=0$, in unit groups of rings.

Throughout the paper $\mathbf{C}, \mathbf{R}_{+}, \mathbf{Z}$ and $\mathbf{N}$ denote the sets of complex, positive real, integer and natural numbers, respectively. By $|a|$ we mean the absolute value of $a \in \mathbf{C}$.

We will investigate torsion free rings with unity. $\mathcal{J}(R)$ denotes the Jacobson radical of a ring $R$. We say that an algebra $A$ over $\mathbf{C}$ admits a trace function if there exists a $\mathbf{C}$-linear map $\operatorname{Tr}: A \longrightarrow \mathbf{C}$ such that $\operatorname{Tr}(a b)=\operatorname{Tr}(b a)$ for $a, b \in A$, $\operatorname{Tr}(e) \in \mathbf{R}_{+}$for all idempotents $e \in A \backslash\{0\}$ and $\operatorname{Tr}(n)=0$ for every nilpotent element $n \in A$.

Let $\langle x, y\rangle$ denote the subgroup of the ring $R$ generated by two units $x, y \in R$. $F_{2}$ stands for the free group on two generators.

Theorem 1. Let $R$ be a torsion free ring. Assume that $a, b \in R, a^{2}=b^{2}=0$ and $a b$ is not nilpotent. Then there exists $m \in \mathbf{N}$ such that $\langle 1+m a, 1+m b\rangle \cong F_{2}$. If

Received by the editors October 15, 1997.

1991 Mathematics Subject Classification. Primary 16U60.

The author was supported by KBN reasearch grant 2P03A 00312.

(C)1999 American Mathematical Society 
moreover $\mathbf{C} \otimes_{\mathbf{Z}} R$ admits a trace function $T r$, then $\langle 1+a, 1+b\rangle \cong F_{2}$, provided that $|\operatorname{Tr}(a b)|>2 \operatorname{Tr}(1)$.

Proof. Let $\mathbf{Z}\langle a, b\rangle$ be the subring of $R$ generated by $a$ and $b$. Denote $A=\mathbf{C} \otimes_{\mathbf{Z}}$ $\mathbf{Z}\langle a, b\rangle$. If there are no relations in $A$ other than those coming from $a^{2}=b^{2}=0$, then $A$ is isomorphic to $\mathbf{C}\left\langle a, b: a^{2}=b^{2}=0\right\rangle$. Hence we can define a homomorphism $\phi: A \longrightarrow M_{2}(\mathbf{C})$ by the rule $\phi(a)=\left(\begin{array}{ll}0 & 2 \\ 0 & 0\end{array}\right), \phi(b)=\left(\begin{array}{ll}0 & 0 \\ 2 & 0\end{array}\right)$ since matrices in the definition of $\phi$ are nilpotent of degree 2. Because $\langle 1+\phi(a), 1+\phi(b)\rangle \cong F_{2}$ by [6], we have also $\langle 1+a, 1+b\rangle \cong F_{2}$. So assume that there exists a nontrivial relation between elements of the set $S$ of nonzero words in $a, b$. Elements of $S$ are of the form $x(a b)^{n} y$ where $n \geq 0, x \in\{1, b\}, y \in\{1, a\}$. $S$ can be ordered by lexicographic order assuming that $a>b$. So there is a relation of the form $w=\sum_{i} \alpha_{i} w_{i}$, where $\alpha_{i} \in \mathbf{C}, w, w_{i} \in S$ and $w>w_{i}$ for all $i$. Note that any word $z \in S$ of length greater than the length of $w$ has to contain $w$ as a subword. Hence substituting $\sum_{i} \alpha_{i} w_{i}$ in place of $w$ we can express $z$ as a linear combination of words which are smaller than $z$. Repeating this argument we can prove that $A=\operatorname{Lin}_{\mathbf{C}}\{z \in S$ : length of $z \leq$ length of $w\}$. In particular $\operatorname{dim}_{\mathbf{C}} A<\infty$. Let

$$
A / \mathcal{J}(A) \cong M_{n_{1}}(\mathbf{C}) \times M_{n_{2}}(\mathbf{C}) \times \cdots \times M_{n_{k}}(\mathbf{C})
$$

First we will prove that $n_{i} \leq 2$ for each $i$. Clearly there exists a homomorphism $\phi_{i}$ : $A \longrightarrow M_{n_{i}}(\mathbf{C})$ which is onto. Since $\phi_{i}(a)^{2}=0$, one obtains $\operatorname{ker} \phi_{i}(a) \supseteq \operatorname{Im} \phi_{i}(a)$. It follows that $n_{i}-\operatorname{dim} \operatorname{Im} \phi_{i}(a) \geq \operatorname{dim} \operatorname{Im} \phi_{i}(a)$ and therefore $\operatorname{rank} \phi_{i}(a) \leq n_{i} / 2$. Define $X=\operatorname{Lin}\left\{\left(\phi_{i}(a) \phi_{i}(b)\right)^{t}: t=0,1, \ldots\right\}$. Since $\operatorname{rank}\left[\phi_{i}(a) \phi_{i}(b)\right] \leq\left[n_{i} / 2\right]$ we get $\operatorname{dim} X \leq\left[n_{i} / 2\right]+1$ by the Cayley-Hamilton theorem. It is easy to see that

$$
M_{n_{i}}(\mathbf{C})=X+\phi_{i}(b) X+X \phi_{i}(a)+\phi_{i}(b) X \phi_{i}(a) .
$$

This implies $n_{i}^{2} \leq 4\left(\left[n_{i} / 2\right]+1\right)$. Hence $n_{i} \leq 2$, as desired. Suppose that $n_{i}=1$ for all $i$. This means that $A / \mathcal{J}(A)$ is commutative. Hence $a b-b a \in \mathcal{J}(A)$ which is nilpotent, say of index $m$. This implies $[(a b-b a) a(a b-b a) b]^{m}=0$ and leading to $(-a b a b a b)^{m}=0$, a contradiction, as $a b$ is assumed not to be nilpotent. Hence there exists $i$ such that $n_{i}=2$ and an onto homomorphism $\phi: A \longrightarrow M_{2}(\mathbf{C})$. Clearly $M_{2}(\mathbf{C})=\mathbf{C}\langle\phi(a), \phi(b)\rangle$. Now $\phi(a)^{2}=0$ and $\phi(a) \neq 0$, hence $\operatorname{rank} \phi(a)=$ 1. Similarly $\operatorname{rank} \phi(b)=1$. If $\phi(a) \phi(b)=0$, then $\operatorname{Im} \phi(b)$ is $\phi(a)$ - and $\phi(b)$ invariant. This gives $M_{2}(\mathbf{C}) \operatorname{Im} \phi(b) \subseteq \operatorname{Im} \phi(b)$, a contradiction. Hence $\phi(a) \phi(b) \neq 0$ and similarly $\phi(b) \phi(a) \neq 0$. Since $\operatorname{rank}[\phi(a) \phi(b)] \leq 1$ and $\phi(a) \phi(b) \neq 0$, we get $\operatorname{rank}[\phi(a) \phi(b)]=1$. This implies

$$
\operatorname{Im}[\phi(a) \phi(b)]=\operatorname{Im} \phi(a), \quad k e r[\phi(a) \phi(b)]=\operatorname{ker} \phi(b) .
$$

Assume that $\phi(a) \phi(b)$ is nilpotent. Then $\operatorname{Im}[\phi(a) \phi(b)]=\operatorname{ker}[\phi(a) \phi(b)]$ implies $\operatorname{Im} \phi(a)=\operatorname{ker} \phi(b)$ and we get $\phi(b) \phi(a)=0$, a contradiction. Hence $\phi(a) \phi(b)$ is not nilpotent and we can find a basis $\left\{v_{1}, v_{2}\right\}$ of $\mathbf{C}^{2}$ such that $\phi(a) \phi(b)=$ $\left(\begin{array}{ll}\lambda & 0 \\ 0 & 0\end{array}\right), \lambda \in \mathbf{C} \backslash\{0\}$. From (1) it follows that $\phi(a)=\left(\begin{array}{ll}p & q \\ 0 & 0\end{array}\right)$ and $\phi(b)=$ $\left(\begin{array}{ll}r & 0 \\ s & 0\end{array}\right)$ for some $p, q, r, s \in \mathbf{C}$. Because $\phi(a)$ and $\phi(b)$ are nilpotent we have $p=r=0$. Now we can find $m \in \mathbf{N}$ such that $|m q|,|m s| \geq 2$. In this case $\langle 1+m \phi(a), 1+m \phi(b)\rangle \cong F_{2}$, whence $\langle 1+m a, 1+m b\rangle \cong F_{2}$. This completes the proof of the first assertion of the theorem. 
Now assume that $A$ admits a trace function $\operatorname{Tr}$ and $|\operatorname{Tr}(a b)|>2 \operatorname{Tr}(1)$. By the first part of the proof we can assume that $\operatorname{dim} A<\infty$. Hence $\mathcal{J}(A)$ is nilpotent. Define $\operatorname{tr}: A / \mathcal{J}(A) \longrightarrow \mathbf{C}$ by the rule $\operatorname{tr}(a+\mathcal{J}(A))=\operatorname{Tr}(a)$ for $a \in A$. Then $t r$ is well defined because if $a \in A$ is nilpotent, then $\operatorname{Tr}(a)=0$. It follows also that $\operatorname{tr}(c)=0$ for all nilpotent elements $c \in A / \mathcal{J}(A)$. Of course $\operatorname{tr}$ is $\mathbf{C}$-linear and $\operatorname{tr}(\bar{a} \bar{b})=\operatorname{tr}(\bar{b} \bar{a})$ for $\bar{a}, \bar{b} \in A / \mathcal{J}(A)$. Moreover if $\bar{e} \in A / \mathcal{J}(A)$ is a nonzero idempotent, then there exists a nonzero idempotent $e \in A$ such that $\bar{e}=e+\mathcal{J}(A)$. This implies $\operatorname{tr}(\bar{e})=\operatorname{Tr}(e) \in \mathbf{R}_{+}$. It follows that $\operatorname{tr}$ is a trace function. By the proof of the first part of the theorem we know that

$$
A / \mathcal{J}(A)=\underbrace{\mathbf{C} \times \cdots \times \mathbf{C}}_{k} \times \underbrace{M_{2}(\mathbf{C}) \times \cdots \times M_{2}(\mathbf{C})}_{l} .
$$

Let $\phi_{i}$ denote the composition of the quotient map $A \longrightarrow A / \mathcal{J}(A)$ with the projection on the $i$-th factor of $A / \mathcal{J}(A)$. Then $\phi_{i}(a)=0$ for $i \leq k$ because $a^{2}=0$ and $\phi_{i}(a)=\left(\begin{array}{cc}0 & q_{i} \\ 0 & 0\end{array}\right)$ for $i>k$. Similarly $\phi_{i}(b)=0$ for $i \leq k$ and $\phi_{i}(b)=\left(\begin{array}{cc}0 & 0 \\ s_{i} & 0\end{array}\right)$ for $i>k$. The trace function $t r$ has the form $t r=\sum_{i} \lambda_{i} t r_{i}, \lambda_{i} \in \mathbf{R}_{+}$, where $t r_{i}$ denotes the usual trace function. In fact, it is easy to verify that the trace function on matrices has to be a scalar multiple of the usual trace function. The condition $|\operatorname{Tr}(a b)|>2 \operatorname{Tr}(1)$ implies $\left|\sum_{i>k} \lambda_{i} q_{i} s_{i}\right|>2\left(\sum_{i \leq k} \lambda_{i}+\sum_{i>k} 2 \lambda_{i}\right)$. Assume that $\left|q_{i} s_{i}\right| \leq 4$ for all $i>k$. This implies

$$
4 \sum_{i>k} \lambda_{i} \leq 2 \sum_{i \leq k} \lambda_{i}+4 \sum_{i>k} \lambda_{i}<\left|\sum_{i>k} \lambda_{i} q_{i} s_{i}\right| \leq \sum_{i>k} \lambda_{i}\left|q_{i} s_{i}\right| \leq 4 \sum_{i>k} \lambda_{i}
$$

a contradiction. Hence we can find $i_{0}$ such that $\left|q_{i_{0}} s_{i_{0}}\right|>4$. It is clear that $\phi_{i_{0}}(1+a)=\left(\begin{array}{cc}1 & q_{i_{0}} \\ 0 & 1\end{array}\right), \phi_{i_{0}}(1+b)=\left(\begin{array}{cc}1 & 0 \\ s_{i_{0}} & 1\end{array}\right)$, or changing the basis $\phi_{i_{0}}(1+a)=$ $\left(\begin{array}{cc}1 & \sqrt{q_{i_{0}} s_{i_{0}}} \\ 0 & 1\end{array}\right), \phi_{i_{0}}(1+b)=\left(\begin{array}{cc}1 & 0 \\ \sqrt{q_{i_{0}} s_{i_{0}}} & 1\end{array}\right)$ where $\left|\sqrt{q_{i_{0}} s_{i_{0}}}\right|>2$. Therefore $\langle 1+a, 1+b\rangle \cong F_{2}$.

Next we will prove a few corollaries concerning rings $R$ with involution $*$ satisfying the condition: $a a^{*}=0 \Longrightarrow a=0$ for $a \in R$. This is the positive-definite condition explored in $[1,2.2]$. There exist many examples of rings of this type: $M_{n}(\mathbf{C})$ with Hermitian conjugation, group rings $\mathbf{C}[G]$ or more generally $\mathbf{C}[S]$ where $S$ is an inverse semigroup and $\left(\sum \alpha_{s} s\right)^{*}=\sum \bar{\alpha}_{s} s^{-1}$; cf. [5, the proof of Lemma 2.3]. Also it is known that many examples of rings of operators on a Hilbert space are of this kind.

By $U(R)$ and $Z(R)$ we denote the group of units and the center of a $\operatorname{ring} R$, respectively.

Corollary 2. Let $R$ be a positive-definite ring. Then $F_{2} \subseteq U(R)$ if either of the following holds:

(1) $R$ contains nonzero nilpotent elements,

(2) $R$ contains noncentral idempotents.

Proof. (1) There exists $a \in R \backslash\{0\}, a^{2}=0$. Because $a a^{*}$ is not nilpotent, Theorem 1 implies that we can find $m \in \mathbf{N}$ such that $\left\langle 1+m a, 1+m a^{*}\right\rangle \cong F_{2}$. This implies $F_{2} \subseteq U(R)$. 
(2) If $e R(1-e)=0$ and $(1-e) R e=0$, then $e r=e r e$ and re $=$ ere for $r \in R$. Hence $e r=r e$ and $e \in Z(R)$, a contradiction. Therefore $e R(1-e) \neq 0$ or $(1-e) R e \neq 0$. Let for example $e R(1-e) \neq 0$. Then we can find $r \in R$ such that $e r(1-e) \neq 0$. Since $[e r(1-e)]^{2}=0$, case (1) applies.

Corollary 3. Let $R$ be a positive-definite prime ring. If $R$ has zero divisors, then $F_{2} \subseteq U(R)$.

Proof. Assume that $a b=0, a, b \in R \backslash\{0\}$. By primeness of $R$ we can find $r \in R$ such that $b r a \neq 0$. Because $(b r a)^{2}=0$, we have $F_{2} \subseteq U(R)$ by Corollary 2 .

Corollary 4. Let $R$ be a positive-definite noncommutative algebra over an uncountable field. Then the multiplicative semigroup $R^{\circ}$ of $R$ contains a free noncommutative subsemigroup.

Proof. If $R$ contains nilpotent elements, then $F_{2} \subseteq U(R)$ by Corollary 2. If $R$ does not contain nilpotent elements, $R$ can be represented as a subdirect product of domains $D_{i}, i \in I$; cf. [1, Theorem 1.1.1]. Because $R$ is noncommutative, we can find $i \in I$ such that $D_{i}$ is noncommutative. By [2] $D_{i}^{\circ}$ contains a noncommutative free semigroup and hence $R^{\circ}$ also.

\section{REFERENCES}

[1] Herstein, I.N. "Rings with involution", Chicago Lect. in Math., The Univ. of Chicago Press, 1976. MR 56:406

[2] Klein, A.A. "Free subsemigroups of domains", Proc. AMS 116(1992), 339-341. MR 92m: 16045

[3] Marciniak, Z.S. and Sehgal, S.K. "Constructing free subgroups of integral group ring units", Proc. AMS 125(1997), 1005-1009. MR 97f:16057

[4] Marciniak, Z.S. and Sehgal, S.K. "Subnormal subgroups of group ring units", Proc. AMS 126(1998), 343-348. MR 98d:16046

[5] Munn, W.D. "Semiprimitivity of inverse semigroup algebras", Proc. Royal Soc. Edinb. 93A(1982), 83-98. MR 84e:20077a

[6] Sanow. I.N. "The property of certain representation of free group", Dokl. AN SSSR 57(1947), 657-659, in Russian.

Institute of Mathematics, University of Warsaw, Banacha 2, 02-097 Warsaw, Poland E-mail address: asalwa@mimuw.edu.pl 